\title{
Mechanical Stimulation-Induced Cross-Adaptation in Plants: An Overview
}

\author{
Zhong-Guang Li $\cdot$ Ming Gong
}

Received: 1 August 2011 /Revised: 15 August 2011 /Accepted: 18 August 2011 /Published online: 3 September 2011

(C) The Author(s) 2011. This article is published with open access at Springerlink.com

\begin{abstract}
Mechanical stimulation (MS), existing widely but ignored usually in nature, is one of the environmental stress factors. MS not only affects growth, development, morphogenesis, and even survival of plants, but also induces the formation of cross-adaptation. In cross-adaptation, plants make use of common pathways and components to adapt to a range of different stresses after exposure to one specific stress. Here, we summarize current knowledge of MS-induced cross-adaptation to chilling, heat, salt, drought, and pathogen stress, as well as its possible mechanisms.
\end{abstract}

Keywords Mechanical stimulation · Cross-adaptation . Calcium messenger system $\cdot$ Reactive oxygen species . Nitric oxide

\section{Introduction}

Plants, due to sessile nature, are constantly exposed to various biotic and abiotic stresses. Abiotic stresses, including extreme temperature, drought, high salinity, heavy metal, and mechanical

\section{Z.-G. Li $(\bowtie)$}

School of Life Sciences, Yunnan Normal University, Kunming 650092, Yunnan, People's Republic of China e-mail: zhongguang_li@163.com

Z.-G. Li $\cdot$ M. Gong

School of Life Sciences, Engineering Research Center of Sustainable Development and Utilization of Biomass Energy, Ministry of Education,

Yunnan Normal University,

Kunming 650092, Yunnan, People's Republic of China

Z.-G. Li $\cdot$ M. Gong

Key Laboratory of Biomass Energy and Environmental Biotechnology, Yunnan Normal University,

Kunming 650092, Yunnan, People's Republic of China stimulation (MS), are major causes of crop failure worldwide. MS, namely mechanical stress, such as wind, raining, hailing, touching, gravity, grazing, loading, and soil obstruction, existing widely but ignored usually in nature, is one of the abiotic factors affecting growth, development, morphogenesis, and even survival of plants (Jaffe and Biro 1979; Knight 2000; Braam 2005; Fig. 1). Plants have developed sensory mechanisms to detect mechanical stimulation and to induce a suite of responses such as anatomical, physiological, biochemical, biophysical, and molecular collectively termed "thigmomorphogenesis" (Jaffe and Biro 1979; Knight 2000; Braam 2005). The study on MS may have been traced back to The Power of Movement in Plants by Charles Darwin (1880), but advances in MS is very slow due to its diversity, complexity, uncertainty, and it is hard to quantify. With the development of molecular biology technique and imaging technique, understanding MS has made great progresses in recent years.

For excellent additional information, we refer readers to wonderful reviews written more recently (Braam 2005; Chehab et al. 2009; Zhang et al. 2010). Here, we summarize current knowledge of MS-induced cross-adaptation to chilling, heat, salt, drought, and pathogen stress, as well as its possible mechanisms.

\section{MS-Induced Cross-Adaptation}

Cross-adaptation, namely cross-tolerance, is the phenomenon by which a plant resistance to a stress leads to resistance to another form of stress. For example, cold acclimation can increase heat tolerance of winter rye, ultraviolet radiation can enhance heat tolerance in cucumber and cold hardiness in Rhododendron, and salt pretreatment can induce cold hardiness rapidly in spinach and potato (Knight 2000). Our previous experiments results showed that heat shock can improve resistance of maize seedlings to heat, chilling, salt, 


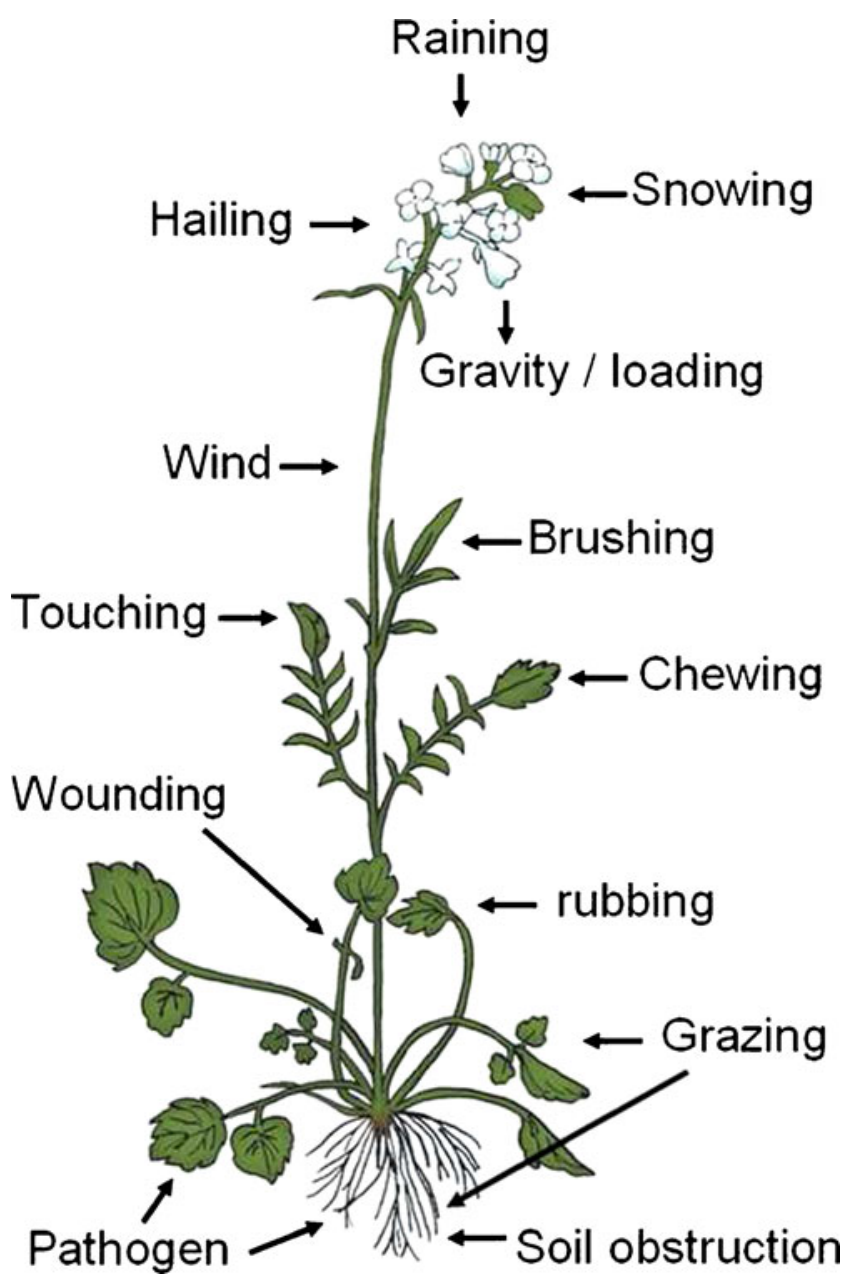

Fig. 1 Types of mechanical stimulation in plants. Plants subject simultaneously and sequentially to various overground and underground mechanical stimulations in nature (adapted in part from Buchanan et al. 2000)

and drought (Gong et al. 2001). We and other authors have found that mechanical stress can induce cross-adaptation in plants as summarized as follows (Table 1).

\section{Chilling Tolerance}

As mentioned above, low temperature is a principal abiotic stress factor affecting growth, development, productivity, geographical distribution, and even survival of plants. Jaffe and Biro (1979) have found that in bean and maize plants, rubbing the stems with paper can increase cold tolerance at sub-zero temperatures than do untreated controls and recover more readily after cold stress, but the mechanisms by which such an effect is controlled are not known.

Keller and Steffen (1995) have investigated the effects of brushing 80 cycles of strokes per day with a wooden bar on the chilling tolerance and metabolism of nonstructural carbohydrates such as soluble sugars and starch in tomato leaves before, during, and after a chilling stress. After the appearance of the second true leaf, tomato plants (Lycopersicon esculentum Mill. cv. Caruso) that was brushed for 15 days or control plants without mechanical stress application are subjected to a 7-day chilling treatment $\left(8 / 5^{\circ} \mathrm{C}\right.$ day/night $)$. The results show that brushing leads to shorter plants with a $34 \%$ reduction in leaf dry weight per area and a 59\% reduction of soluble sugars and starch. A greater chilling tolerance in the brushed plants is demonstrated by the maintenance of a significantly higher photosystem II efficiency in brushed plants compared to that of the control plants after 7 days of chilling treatment, less visible damage to the leaf tissue, and a more rapid resumption of growth during 3 days of recovery as compared to control plants. In addition, during the chilling treatment, contents of soluble sugars per leaf dry weight increase 15 -fold in the brushed plants and fivefold in control plants. These results demonstrate that brushing can increase chilling tolerance in tomato plants. The observed differences in chilling tolerance and contents of soluble sugars in the leaves may indicate an involvement of soluble sugar levels in acclimation to chilling.

We use MS by increasing the rotational speed of rotary shaker, relative quantified MS, and have found that MS can alleviate decrease in vitality, that is, triphenyl tetrazolium chloride reduction, of tobacco suspension-cultured cells and reduce accumulation of indicator of lipid peroxidation, malonaldehyde (MDA) under chilling stress at $1{ }^{\circ} \mathrm{C}$, which in turn improve survival percentage under chilling stress and regrowth ability of tobacco suspension cells during normal culture condition after chilling stress. Interestingly, MS can increase the

Table 1 Example of types of MS-induced cross-adaptation in plants

\begin{tabular}{llll}
\hline Types of MS & Species & Cross-adaptation & References \\
\hline Brushing & Bean, Maize, tomato & Chilling, drought & Keller and Steffen (1995); Jaffe and Biro (1979) \\
Pressing & Cucumber & Pathogen & Wang et al. (2006); Zhao et al. (2005a, b) \\
Wounding & Tomato, Arabidopsis & Salt, abiotic and biotic stress & Capiati et al. (2006); Walley et al. (2007) \\
Wind & Bean & Pest & Jaffe and Biro (1979); Cipollini (1997) \\
Increasing rotational speed & Tobacco & Heat, chilling, salt, heavy metal & Li and Gong (2008, 2009, 2010, 2011); \\
& & & Li et al. (2009, 2011) \\
\hline
\end{tabular}


activity of $\Delta^{1}$-pyrroline-5-carboxylate synthetase (P5CS), a key enzyme of proline biosynthesis in glutamate pathway, and induce accumulation of endogenous proline in tobacco cells. Exogenously applied optimal concentration of proline to cells also can enhance its endogenous level in normal culture condition and survival percentage of the cells under chilling stress. These results suggest that MS can improve chilling tolerance of tobacco suspension cells and this improvement is related to proline (Li and Gong 2011).

\section{Heat Tolerance}

With the development of global warming, high temperature has already become a noticeable abiotic stress factor limiting crop yield. Many groups have been concentrating on heat tolerance in plant, especially crop plants. In tobacco suspension-cultured cells, we have found that mechanical stimulation by increasing rotational speed in a shaker incubator can increase survival percentage under heat stress at $43^{\circ} \mathrm{C}$ and regrowth ability of tobacco suspension cells during recovery after heat stress, and alleviate the loss of cell vitality and accumulation of MDA in tobacco suspension cells ( $\mathrm{Li}$ and Gong 2008). Interestingly, MS can enhance the activity of cell wall peroxidase, one of the enzyme resources of hydrogen peroxide $\left(\mathrm{H}_{2} \mathrm{O}_{2}\right)$ production in plant cells; induce $\mathrm{H}_{2} \mathrm{O}_{2}$ accumulation; and increase $\mathrm{pH}$ value of culture medium in tobacco suspension cells. The pretreatments with peroxidase inhibitors, $\mathrm{KCN}$ or salicylhydroxamic acid, respectively, inhibit sharply MS-induced $\mathrm{H}_{2} \mathrm{O}_{2}$ burst in tobacco suspension cells and increase $\mathrm{pH}$ value in the culture medium ( $\mathrm{Li}$ and Gong 2009). In addition, exogenously applied optimal concentration of $\mathrm{H}_{2} \mathrm{O}_{2}$ to cells also can enhance survival percentage of cells under heat stress (Li and Gong 2008). Furthermore, the production of MS-induced oxidative burst in tobacco suspension cells can be strengthened by addition of exogenous $\mathrm{Ca}^{2+}$, while weakened significantly by application of $\mathrm{Ca}^{2+}$ chelator ethylene glycol-bis(baminoethylether)- $\mathrm{N}, \mathrm{N}, \mathrm{N}^{\prime}, \mathrm{N}^{\prime}$-tetraacetic acid (EGTA), plasma membrane $\mathrm{Ca}^{2+}$ channel blocker $\mathrm{La}^{3+}$, intracellular $\mathrm{Ca}^{2+}$ channel blocker ruthenium red (RR), as well as calmodulin antagonists chlorpromazine (CPZ) and trifluoperazine (TFP), respectively (Li and Gong 2010).

In addition to these, we also found that MS obviously can enhance calmodulin (CaM) activity in tobacco suspension cells, the MS-induced heat tolerance in tobacco suspension cells is markedly strengthened by addition of exogenous $\mathrm{Ca}^{2+}$, but weakened significantly by application of $\mathrm{Ca}^{2+}, \mathrm{La}^{3+}, \mathrm{RR}$, as well as CPZ and TFP, respectively ( $\mathrm{Li}$ and Gong 2009). These results suggest that MS could induce heat tolerance of tobacco suspension cells and the cross-talk between calcium messenger system and $\mathrm{H}_{2} \mathrm{O}_{2}$ is involved in the acquisition of this heat tolerance.

\section{Salt Tolerance}

Excess soil salt causes injury to plant due to osmotic stress, ion toxicity, and nutrient deficiency is referred to as salt stress. Soil salinity is a major abiotic stress in plant agriculture worldwide. This has led to research into salt tolerance with the aim of improving crop plants. It has previously been shown that salt stress causes the activation of other wound-related genes in tomato plants (Solanum lycopersicum) and the accumulation of proteinase inhibitors. In the work of Capiati et al. (2006), it is shown that mechanical wounding by cutting with a dented forceps increases salt stress tolerance in tomato plants through a mechanism that involves the signaling peptide system and the synthesis of jasmonic acid. The results are also provided indicating that calmodulin-like activities are necessary for the downstream signaling events that lead to cross-tolerance between wounding and salt stress. Finally, evidence is gathered supporting the hypothesis that LeCDPK1, a $\mathrm{Ca}^{2+}$-dependent protein kinase from tomato, could participate in this cross-tolerance mechanism interrelating the signaling responses to wounding and salt stress.

In our previous work, it is clearly shown that tobacco suspension cells subjected to mechanical stimulation by increasing rotational speed in a shaker incubator can increase survival percentage under salt and heavy metal chromium stress and regrowth ability of tobacco suspension cells during recovery in normal culture condition after stress, and alleviate the loss of cell vitality and accumulation of marker of lipid peroxidation, MDA, of tobacco suspension cells ( $\mathrm{Li}$ et al. 2011). These results suggest that mechanical stimulation can induce the acquisition of salt and heavy metal tolerance in tobacco suspension cells, but its tolerance mechanism needs further study.

\section{Resistant to Pathogen}

Biotic stress such as bacteria, fungus, and virus, is another stress factor affecting plant growth, development, and yield. During the course of pathogens penetrating the plant cell, besides chemical secretion, the pathogens may cause mechanical signal by the physical pressure on the plant cell. Zhao et al. (2005a) use the stress by pressing with a certain weight on the leaf surface as the mechanical signal elicitor to find the effect of plant resistance induced by stress. The results show that suitable stress stimulation can significantly improve the resistance of plant pathogen. However, disruption on the plasma membrane-cell wall adhesion will absolutely eliminate this kind of inducement effect, which suggests that the plant resistance inducement by stress depend on the adhesion of plasma membrane-cell wall. Also, the results found that stress stimulation may 
lead to synthesis of lignin and obviously increase the activity of phenylalanine ammonia lyase (PAL), chitinase, and beta-1,3-glucanase. The results illustrate that stress stimulation may enhance ability of the plant cell resistant to pathogen penetration through eliciting the accumulation of pathogens suppression or antimicrobial chemical substance in the plant cell. In addition, Wang et al. (2006) have investigated plant resistance and the variation of antidisease enzyme activity in cucumber leaves after mechanical stress loading and found that the appropriate mechanical stimulation can significantly improve plant resistance, alter the activity of PAL and peroxidase (POD), and induce synthesis of lignin. Furthermore, data show that effects of stress on these cellular fundamental events are eliminated when the adhesion between plasma membrane and cell wall is disrupted.

In the nonhost resistance, both the chemical signal and the mechanical stress signal are considered to have contribution to the entire defense reaction acted by the plant. Zhao et al. (2005b) have found that, as a mechanical signal elicitor, the appropriate stress stimulus is proved to effectually induce the resistance of cucumber seedling to Cladosporium cucumerinum. The activities of resistancerelated enzymes such as PAL and POD were significantly increased after the treatment of the stress stimulus on leaves. Also, the results show that stress stimulation may cause synthesis of lignin, which acts as the physical barrier to defend the pathogens. Gus-Mayer et al. (1998) substitute the penetrating fungus by local mechanical stimulation by using a needle of the same diameter as a fungal hypha, by local application of a structurally defined fungus-derived elicitor, or by a combination of the two stimuli. The results have found that the local mechanical stimulus alone induce the translocation of cytoplasm and nucleus to the site of stimulation, trigger the accumulation of intracellular reactive oxygen species (ROS), and induce the expression of elicitor-responsive genes such as $P R 1, P R 2$, and WRKY1 similar to the fungal infection hypha. In addition, the elicitor is applied locally to the cell surface without mechanical stimulation, morphological changes are not detected but intracellular ROS also accumulate rapidly. However, a combination of the mechanical stimulus with simultaneous application of low doses of elicitor closely simulated early reactions to fungal infection including cytoplasmic aggregation, nuclear migration, and reactive oxygen species accumulation. These results suggest that mechanical stimulation by the invading fungus is responsible for the observed intracellular rearrangements and may trigger some of the previously demonstrated changes in the activity of elicitor-responsive genes, whereas chemical stimulation is required for additional biochemical processes.
Drought Tolerance

Drought can be defined as the absence of rainfall or irrigation for a period of time sufficient to deplete soil moisture and injure plants. Drought stress results when water loss from the plant exceeds the ability of the plant's roots to absorb water and when the plant's water content is reduced enough to interfere with normal plant processes. Jaffe and Biro (1979) have found that rubbing the stem with paper or stroking the shoot with the hand can enhance the ability of bean and maize to recover from drought. Leaves detached from stroked plants lose water less rapidly than leaves from untreated ones. Brushed seedlings lose less water per plant but more water per unit leaf area than controls. In addition, studies with lettuce suggest that there is a greater density of stomata on the leaf surface of the brushed plants than on the controls, although the number of stomata per leaf is not significantly different between the two. Furthermore, the results show an increased conductance to water vapor in wind-exposed or continuously shaken Festuca arundinacea compared to untreated controls. On the other hand, it has been found that brushed lettuce, cauliflower, and celery seedlings with paper recover less well from drought than unbrushed controls. Although it is suggested that this may have been due to epidermal abrasion caused by the wind, as with lettuce there are more, albeit smaller stomata per unit leaf area in wind-exposed plants, than those kept in still air. However in shaken plants, in which abrasion is unlikely to have occurred, the density and size of stomata were similar to those of the control plants.

A large body of literature addresses stoma regulation by the plant hormone abscisic acid (ABA), which accumulates during wilting and reduces water loss by closing stomata. Interestingly, in Phaseolus vulgaris plants, the content of ABA increased threefold after brushing with paper. In addition to this, tomato plants have been found to wilt for short periods as a result of brushing (Jaffe and Biro 1979).

\section{Other Stress Tolerance}

As discussed above, induction of enzymes such as PAL, POD, and the accumulation of such phenolics as lignin can occur in response to pathogen attack, mechanical stimulation, and are thought to function in the resistance of plants to damage by these stresses. It is one of the most universal biochemical stress responses in plants that the phenylpropanoid pathway is activated by various environmental stimuli. Cipollini (1997) has investigated whether induction of components of this generalized stress response by wind-induced mechanical stimulation can influence the resistance to pests of common bean. In greenhouse studies, 7- to 10-day-old bean seedlings 
exposed to daily periods of fan-produced wind cause increase in activities of POD and cinnamyl alcoholdehydrogenase and enhance the accumulation of lignin in primary leaves of these plants. Interestingly, egg production and population growth of two-spotted spider mites are reduced when offered leaves of mechanically stimulated plants in leaf disk and whole-plant bioassays. Infection by anthracnose after inoculation in a detached leaf bioassay is also reduced in leaves of mechanically stimulated plants. The consistent positive association between the enhanced activity of the lignin branch of the phenylpropanoid pathway and enhance resistance to pests found in leaves of mechanically stimulated plants, which illustrates one way in which exposure of plants to environmental stimuli that activate a generalized stress response such as wind can influence the interactions of those plants with other environmental stimuli like pests.

\section{Possible Mechanisms of MS-Induced Cross-Adaptation}

How plants sense external signal and transduce it into the cells is primary research field in MS-induced signal transduction. Knight (2000) have found that MS by touch with glass bar can rapidly trigger accumulation of $\mathrm{Ca}^{2+}$ in cytoplasm within several seconds, which in turn induces expression of $\mathrm{TCH}$ genes encoding $\mathrm{CaM}$, calmodulin-like proteins (CML), and xyloglucan endotransglucosylase/ hydrolase (Braam and Davis 1990; Braam 2005; Lee et al. 2005). In the study of Monshuasen et al. (2009), they have identified that concentration of $\mathrm{Ca}^{2+}$ in cytoplasm is increased by mechanical force, namely $\mathrm{Ca}^{2+}$ signature. MS inducing $\mathrm{Ca}^{2+}$ signature is decoded by CaM, CML, calcium-dependent protein kinase, or other calcium-binding protein, and triggers downstream biological events as well as responses to MS.

ROS, including $\mathrm{H}_{2} \mathrm{O}_{2}$, superoxide anion free radical $\left(\mathrm{O}_{2}{ }^{-}\right)$, hydroxyl radical $(\mathrm{OH})$, is another second messenger in plant cells, especially in adversely environmental conditions. In normal conditions, production and removal of ROS are homeostasis, that is, ROS keeps a very low, nontoxic physical level in plant cells. When plants are subjected to environmental stress such as extreme in temperature, drought, high salinity, and MS, production of ROS is more than its removal and lead to rapid accumulation in different compartment of the cell and produce oxidative stress (Ahmad et al. 2008). As discussed earlier, data from our laboratory have showed that MS can induce $\mathrm{H}_{2} \mathrm{O}_{2}$ burst in tobacco suspension cells and this oxidative burst can be strengthened by exogenous $\mathrm{Ca}^{2+}$ treatment, while weakened significantly by application of EGTA, $\mathrm{La}^{3+}, \mathrm{RR}$, as well as CaM antagonists CPZ and TPF, respectively (Li and Gong 2008, 2009, 2010).
These results suggest that calcium messenger system involves in MS-induced $\mathrm{H}_{2} \mathrm{O}_{2}$ burst in tobacco suspension culture cells. Han and Yuan (2004) also have found that generation of active oxidative species is induced by shear stress in suspension cultures of Taxus cuspidata. Additionally, inhibition studies with diphenylene iodonium and azide suggest that the key enzyme responsible for oxidative bursts under the shear stress is primarily nicotinamide adenine dinucleotide phosphate-oxidase and the contribution of peroxidase for oxidative bursts was less. Furthermore, oxidative bursts elicited by the shear stress are suppressed by treatment with suramin, nifedipine, and neomycin prior to the shear stress treatment suggesting that G-protein, $\mathrm{Ca}^{2+}$ channel, and phospholipase $\mathrm{C}$ are involved in the signal pathway for oxidative bursts induced by the shear stress. Similarly, nitric oxide (NO), another gaseous molecular in plant cells, plays a very important role in plant growth, development, and the formation of stress tolerance. Mechanical stimulation by centrifugation can improve accumulation of $\mathrm{NO}$ in Arabidopsis and elicit DNA fragment (Garces et al. 2001). Our previous work also have identified that MS by increasing rotational speed of a rotary shaker can rapidly accumulate NO in tobacco suspension-cultured cells, and this accumulation is regulated by $\mathrm{Ca}^{2+}$ and $\mathrm{CaM}$ through controlling activity of nitric oxide synthase activity (Ke et al. 2011).

Walley et al. (2007) considers that physical stress is a mechanism common to mounting a response against a broad range of environmental stresses; they use mechanical wounding as the stress stimulus and perform whole genome microarray analysis of Arabidopsis thaliana leaf tissue. This causes the identification of a number of rapid wound responsive (RWR) genes. Comparison of RWR genes with published abiotic and biotic stress microarray datasets demonstrates a large overlap across a wide range of environmental stresses. Interestingly, RWR genes also exhibit a striking level and pattern of circadian regulation with induced and repressed genes displaying antiphasic rhythms. In addition, using bioinformatic analysis, they have identified a novel motif overrepresented in the promoters of RWR genes, herein designated as the rapid stress response element.

Almost all stresses induce the production of a group of proteins called heat shock proteins (Hsps) or stress-induced proteins. According to their approximate molecular weight, these proteins can be divided into five classes in plants: (1) Hsp100, (2) Hsp90, (3) Hsp70, (4) Hsp60, and (5) small heat shock proteins (sHsps) (Wang et al. 2004). Higher plants have at least $20 \mathrm{sHsps}$ and there might be 40 kinds of these sHsps in one plant species. It is believed that this diversification of these proteins reflects an adaptation to various environmental stresses. Major heat shock proteins 
Fig. 2 Possible mechanisms of MS-induced cross-adaptation in plants. MS activates perceptors in cell wall-plasma membrane-skeleton system and triggers production of second messenger such as $\mathrm{Ca}^{2+}$, ROS, and NO, which in turn alters gene expression, leads to change in membrane fluidity, induces synthesis of osmolyte, defense substance, and stress protein, and eventually induces the formation of cross-adaptation

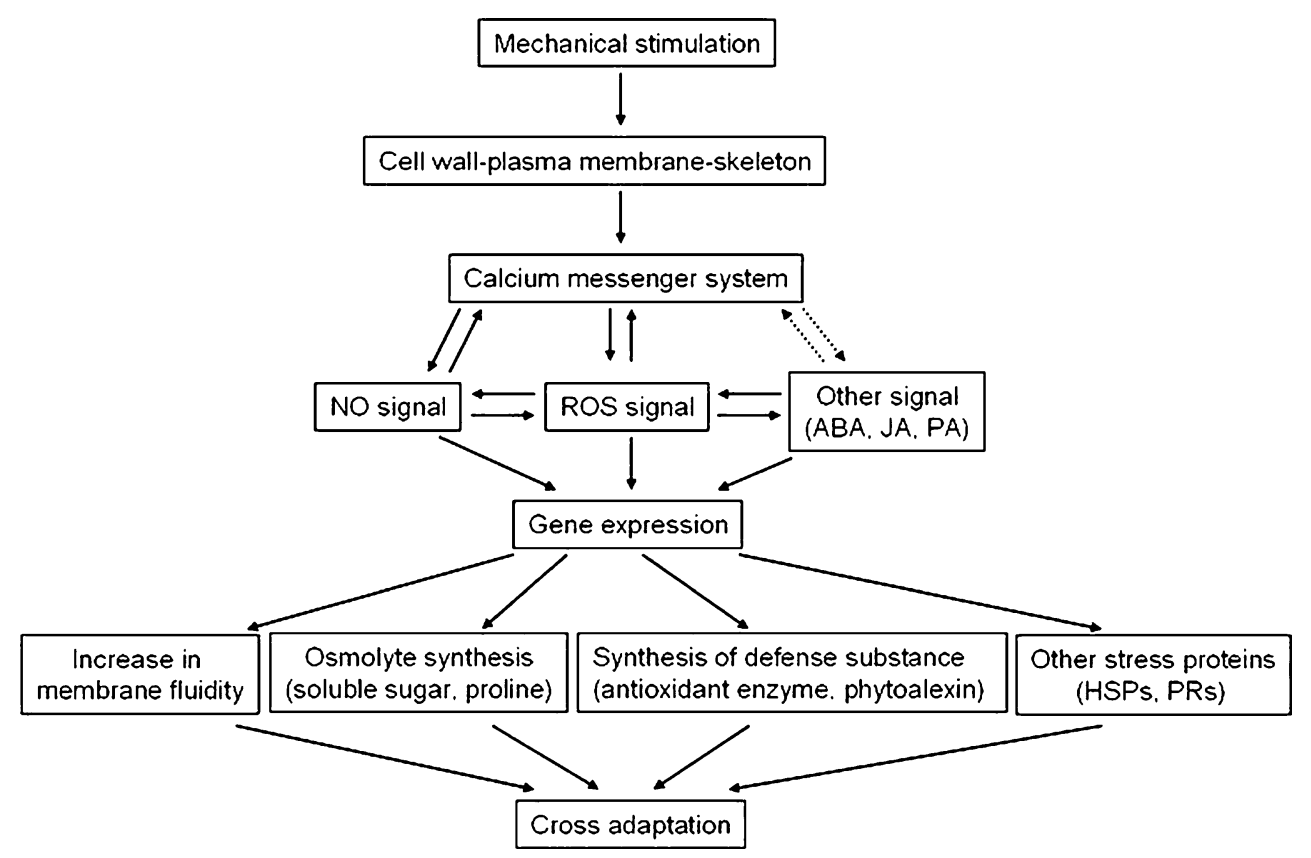

have some kind of related roles in solving the problem of misfolding and aggregation as well as their role as chaperones (Wang et al. 2004). Cheng and Yuan (2009) have found that shear stress not only can induce the release of antioxidant enzyme POD, glutathione reductase (GR), glutathione peroxidase, glutathione-S-transferase (GST) in suspension cultures of Taxus cuspidate but also induce accumulation of HsP70, Hsp80, Hsp90, which in turn trigger adaptation to shear stress.

Lipid is not only a signal involved in mechanotransduction processes in response to shear stress, it also affects membranes fluidity of the plant cells. To understand the responses of phospholipids in shear stress-induced mechanotransduction, a lipidomic approach is employed to profile phospholipid species of T. cuspidata cells under laminar shear stress. The research of Hand and Yuan (2009) have revealed that shear stress can enhance the activation of phospholipase D and phospholipase C compared with control cells and consequently increase PA content in shear stress-induced T. cuspidata cells. In addition, lipidomic analysis reveals, compared with $T$. cuspidata cells cultured in shake flask, the amounts of total PA and PC increase, whereas the amounts of total PG, $\mathrm{PE}$, and PC decline significantly. In contrast, the amount of PS is not significantly different. Furthermore, shear stress also can enhance the ratio of unsaturated to saturated lipid, which in turn improved membrane fluidity of $T$. cuspidata cells and adaptation to this mechanical stimulation (Han and Yuan 2009).

Proline accumulates in many plant species in response to environmental stress, it has multiple functions such as osmotic adjustment, ROS-scavenging, redox-buffering, energy status, as well as acts as small molecular chaperone and plant development signal (Szabados and Savoure 2010; Trovato et al. 2008). As discussed earlier, MS by increasing rotational speed of rotary shaker can increase the activity of P5CS and induce accumulation of endogenous proline in tobacco cells, which in turn improve chilling tolerance ( $\mathrm{Li}$ et al. 2011). Keller and Steffen (1995) have found that brushing with a wooden bar can enhance accumulation of soluble sugars under chilling stress and the concentration of soluble sugars in the leaves may involve in acclimation to chilling. Proline can act as a signaling molecule to modulate mitochondrial functions, influence cell proliferation or cell death, and trigger specific gene expression, which can be essential for plant recovery from stress (Szabados and Savoure 2010; Trovato et al. 2008).

\section{Concluding Remarks}

In conclusion, all environmental stresses including MS lead to common injury mechanisms such as biomembrane damage, osmotic stress, oxidative stress, and protein denaturation. During recovery from stress, plants adopt corresponding measures like maintaining integrality of biomembrane, osmotic adjustment, strengthening of antioxidant system, and producing of stress proteins. When plants are subjected to various mechanical stimulations, which are perceived by same or different sensors may be located in cell wall-plasma membrane-skeleton system and trigger production of second messenger such as $\mathrm{Ca}^{2+}$, ROS, and NO, existing cross-talk among each other, which in turn alter gene expression like $R W R, T C H$, and $P R$, lead to increase in membrane fluidity due to enhancement of the ratio of unsaturated to saturated lipid, induce synthesis of 
osmolytes (soluble sugar, proline), antioxidant enzyme POD, GST, GR, as well as other stress proteins (Hsps, PRs), and eventually trigger the formation of cross-adaptation (Fig. 2). Due to diversity, complexity, compositionality, and hard to quantify MS, however, the mechanisms of signal transduction and cross-adaptation in MS needs in-depth research.

Acknowledgments This research is supported by the Education Department Foundation of Yunnan Province of China (09Y0145).

Open Access This article is distributed under the terms of the Creative Commons Attribution Noncommercial License which permits any noncommercial use, distribution, and reproduction in any medium, provided the original author(s) and source are credited.

\section{References}

Ahmad P, Sarwat M, Sharma S (2008) Reactive oxygen species, antioxidants and signaling in plants. J Plant Biol 51:167-173

Braam J (2005) In touch: plant responses to mechanical stimuli. New Phytol 165:373-389

Braam J, Davis RW (1990) Rain-, wind-, and touch-induced expression of calmodulin and calmodulin-related genes in Arabidopsis. Cell 60:357-364

Buchanna BB, Gruissem W, Jones RL (2000) Biochemistry and molecular biology of plants. American Society of Plant Physiologists, Rockville, MD

Capiati DA, Pais SM, Tellez-Inon MT (2006) Wounding increases salt tolerance in tomato plants: evidence on the participation of calmodulin-like activities in cross-tolerance signaling. J Exp Bot 57:2391-2400

Chehab EW, Eich E, Braam J (2009) Thigmomorphogenesis: a complex plant response to mechano-stimulation. J Exp Bot 60:43-56

Cheng JS, Yuan YJ (2009) Release of proteins: insights into oxidative response of Taxus cuspidata cells induced by shear stress. J Mol Catal B: Enzymatic 58:84-92

Cipollini DF Jr (1997) Wind-induced mechanical stimulation increases pest resistance in common bean. Oecologia 111:84-90

Darwin C (1880) The power of movement in plants. William Clowes, London

Garces H, Durzan D, Pedroso MC (2001) Mechanical stress elicits nitric oxide formation and DNA fragmentation in Arabidopsis thaliana. Annal Bot 87:567-574

Gong M, Chen B, Li ZG, Guo LH (2001) Heat-shock-induced cross adaptation to heat, chilling, drought and salt stress in maize seedlings and involvement of $\mathrm{H} 2 \mathrm{O} 2$. J Plant Physiol 158:1125-1130

Gus-Mayer S, Naton B, Hahlbrock K, Schmelzer E (1998) Local mechanical stimulation induces components of the pathogen defense response in parsley. Proc Natl Acad Sci USA 95:8398-8403

Han RB, Yuan YJ (2004) Oxidative burst in suspension culture of Taxus cuspidata induced by a laminar shear stress in short-term. Biotechnol Progr 20:507-513

Han PP, Yuan YJ (2009) Lipidomic analysis reveals activation of phospholipids signaling in mechanotransduction of Taxus cuspidate cells in response to shear stress. FASEB J 23:623-630

Jaffe MJ, Biro R (1979) Thigmomorphogenesis: the effect of mechanical perturbation on the growth of plants with special reference to anatomical changes, the role of ethylene, and interaction with other environmental stresses. In: Staples R, Mussell H (eds) Stress physiology of crop plants. Wiley, New York

Ke X, Li ZG, Liu X, Gong M (2011) Pathways of mechanical stimulation-induced nitric oxide production and their relationship with $\mathrm{Ca}^{2+}$ and calmodulin in tobacco (Nicotiana tobacum L.) suspension culture cells. Plant Physiol J 47:85-90

Keller E, Steffen KL (1995) Increased chilling tolerance and altered carbon metabolism in tomato leaves following application of mechanical stress. Physiol Plant 93:519-525

Knight H (2000) Calcium signaling during abiotic stress in plants. Int Rev Cytol 195:269-324

Lee D, Polisensky DH, Braam J (2005) Genome-wide identification of touch-and darkness-regulated Arabidopsis genes: a focus on calmodulin-like and XTH genes. New Phytol 165:429-444

Li ZG, Gong M (2008) Mechanical stimulation-induced heat tolerance of suspension cultured cells in tobacco (Nicotiana tabacum L.) and its relation to $\mathrm{H}_{2} \mathrm{O}_{2}$. Plant Physiol Commun 44:41-44

Li ZG, Gong M (2009) Involvement of calcium and calmodulin in mechanical stimulation-induced heat tolerance in tobacco (Nicotiana tabacum L.) suspension cultured cells. Plant Physiol Commun 45:363-365

Li ZG, Gong M (2010) Regulation of calcium messenger system on mechanical stimulation-induced $\mathrm{H}_{2} \mathrm{O}_{2}$ burst in tobacco (Nicotiana tabacum L.) suspension culture cells. Plant Physiol Commun 46:135-138

Li ZG, Gong M (2011) Mechanical stimulation-induced chilling tolerance in tobacco (Nicotiana tabacum L.) suspension culture cells and its relation to proline. Russ J Plant Physiol (in press)

Li ZG, Gong M, Chen XJ (2009) Involvement of cell wall peroxidase in mechanical stimulation-induced oxidative burst in tobacco (Nicotiana tabacum L.) suspension culture cells. Plant Physiol Commun 45:111-114

Li ZG, Yan SZ, Xie H (2011) Effect of mechanical stimulation on salt and heavy metal tolerance in tobacco suspension cultured cells. J Yunnan Norm Univ Nat Sci 31:16-20

Monshuasen GB, Bibikova TN, Weisensee MH, Gilroy S (2009) $\mathrm{Ca}^{2+}$ regulates reactive oxygen species production and $\mathrm{pH}$ during mechanosensing in Arabidopsis roots. Plant Cell 21:2341-2356

Szabados L, Savoure A (2010) Proline: a multifunctional amino acid. Trends Plant Sci 15:89-97

Trovato M, Mattioli R, Costantino P (2008) Multiple roles of praline in plant stress tolerance and development. Rendicnti Lincei 19:325-346

Walley JW, Coughlan S, Hudson ME, Covington MF, Kaspi R, Banu G, Harmer S, Dehesh K (2007) Mechanical stress induces biotic and abiotic stress responses via a novel cis-element. PLoS Gene 3:1800-1812

Wang WX, Vinocur B, Shoseyov O, Altman A (2004) Role of plant heat-shock proteins and molecular chaperones in the abiotic stress response. Trends Plant Sci 9:244-252

Wang BC, Wang JB, Zhao HC, Zhao H (2006) Stress induced plant resistance and enzyme activity varying in cucumber. Colloids Surfaces B: Biointerfaces 48:138-142

Zhang FF, Li ZG, Du CK, Gong M (2010) Responses and signaling transduction of plants to mechanical stimulation. Plant Physiol Commun 46:517-528

Zhao H, Wang BC, Zhao HC, Wand JB (2005a) Stress stimulus induced resistance to Cladosporium cucumerinum in cucumber seeding. Colloids Surfaces B: Biointerfaces 44:36-40

Zhao HC, Zhao H, Wang JB, Wang BC, Wang YN (2005b) Stress stimulation induced resistance of plant. Colloids Surfaces B: Biointerfaces 43:174-178 